\title{
Innovation Qualité 2018 - Die Gewinner (2)
}

\section{Michelle Gerbera; Fabienne Hohl}

a lic. phil. hum. wissenschaftliche Mitarbeiterin FMH, Abteilung Daten, Demographie und Qualität DDQ / Schweizerische Akademie für Qualität in der Medizin SAQM; ' Abteilung Kommunikation der FMH

Die Innovation Qualité, der neue Qualitätspreis der Schweizerischen Akademie für Qualität in der Medizin SAQM der FMH, wurde erstmals am 17. April 2018 vergeben. In der Ausgabe 18 der Schweizerischen Ärztezeitung haben wir die Gewinner der Kategorien Patientenversorgung neu gedacht und Patientensicherheit vorgestellt, heute präsentieren wir die beiden Gewinner der Kategorie Ärzteorganisationen.

\section{Klug entscheiden für mehr Lebensqualität}

Prof. Dr. med. Jean-Michel Gaspoz ${ }^{a}$; Bernadette Häfliger Berger ${ }^{b}$

a Präsident smarter medicine - Choosing Wisely Switzerland; ${ }^{b}$ Geschäftsführerin smarter medicine - Choosing Wisely Switzerland

Vor rund vier Jahren hat die Schweizerische Gesellschaft für Allgemeine Innere Medizin (SGAIM) die Initiative smarter medicine initiiert, welche sich am Vorbild der Choosing-Wisely-Offensive der amerikanischen Ärzteschaft orientiert. In deren Rahmen erarbeitete und veröffentlichte die SGAIM 2014 und 2016 als erste medizinische Fachgesellschaft in der Schweiz sogenannte Top-5-Listen mit medizinischen Massnahmen im ambulanten und stationären Bereich. Darin sind Behandlungen aufgeführt, bei denen eine Über- oder Fehlversorgung festzustellen ist. Auf diese Weise sollen Ärztinnen, Ärzte, Patientinnen und Patienten sensibilisiert werden
: Sie sollen möglichst auf Anwendungen verzichten, welche wenig oder gar keinen Nutzen für die Betroffenen haben, aber deren Lebensqualität beeinträchtigen.

\section{Breite Allianz gegen Überversorgung}

Im Sommer 2016 entwickelte die SGAIM zusammen mit der Schweizerischen Akademie für Medizinische Wissenschaften (SAMW) die Idee eines breiten Trägervereins für smarter medicine. Damit sollte eine umfassende Allianz zwischen Ärzteschaft, nichtärztlichen Gesundheitsberufen, Patientinnen und Patienten so-
Korrespondenz: FMH / Abteilung DDQ Elfenstrasse 18 CH-3000 Bern 15 Tel. 0313591111 saqm[at]fmh.ch
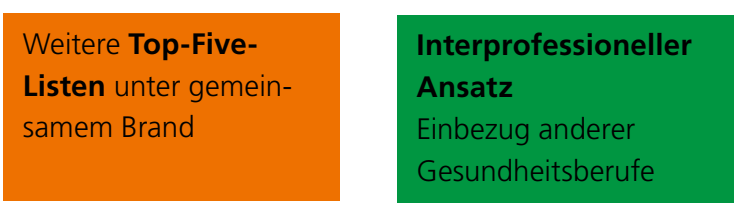

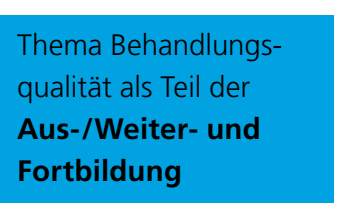
Sensibilisierung der Patient/-innen und Konsument/
innen


Korrespondenz:

Prof. Dr. med.

Jean-Michel Gaspoz

Médecine de premier

recours

Rue Gabrielle-Perret-Gentil 4

CH-1205 Genève

Tel. 0223729520

jean-michel.gaspoz

[at]STOP-SPAM.hcuge.ch

Bernadette Häfliger Berger Schweizerische Gesellschaft für Allgemeine Innere Medizin

Monbijoustrasse 43

CH-3001 Bern

Tel. 0313704006

bernadette.haefliger

[at]sgaim.ch wie Konsumierenden geschaffen werden. Anfang Juni 2017 konnte der Verein smarter medicine - Choosing Wisely Switzerland gegründet werden.

\section{Kommunikation zwischen Betroffenen und Leistungserbringenden}

Ein zentrales Anliegen des Vereins ist, dass die Empfehlungen von den Fachgesellschaften selber erarbeitet und nicht von politischen Behörden oder Krankenversicherern erlassen werden. Die weiteren Ziele wurden gemeinsam mit allen Gründungsmitgliedern erarbeitet (vgl. Abb. 1)

\section{Erste Erfolge und Ausblick}

Im Zuge der Vereinsgründung veröffentlichten verschiedene weitere Fachgesellschaften ihre eigenen Top-5-Listen. Zudem sind in nächster Zeit die Publikationen weiterer Listen geplant.

Vom 1.-3. Oktober 2018 organisiert die SGAIM zusammen mit dem Trägerverein in Zürich den internationalen Choosing-Wisely-Kongress, an dem Expertinnen und Experten aus der ganzen Welt die neuesten Erkenntnisse zum Thema präsentieren werden.

Schliesslich bereitet der Verein eine mehrjährige Sensibilisierungskampagne vor, welche das Thema der Fehl- und Überversorgung der Öffentlichkeit zugäng-

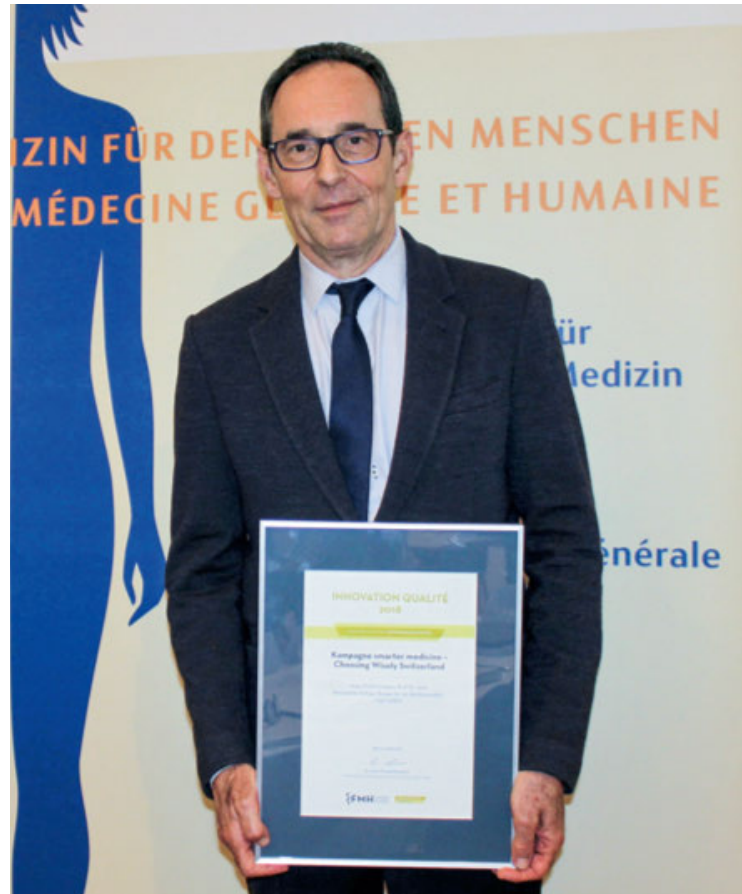

Jean-Michel Gaspoz mit dem Gewinner-Zertifikat.

lich machen soll. Im Rahmen dieser Kampagne sind unter anderem Informationstage und spezielle Informationsmittel für Patientinnen und Patienten geplant. Weitere Informationen zur Initiative smarter medicine stehen auf www.smartermedicine.ch zur Verfügung.

\section{Zertifizierung in der Onkologie - ein Projekt der SGMO}

\section{Walter Mingrone ${ }^{a}$, Christoph Renner ${ }^{b}$, Ursula Kappc, Volker Kirchner ${ }^{\text {, }}$, Thomas von Briele, Kurt Beretta ${ }^{\mathrm{f}}$, Rudolf Morant ${ }^{\mathrm{g}}$, Markus Borner ${ }^{\mathrm{h}}$}

${ }^{a}$ Dr. med., Vorstandsmitglied SGMO und Co-Präsident Swiss Cancer Network (SCN); b Prof. Dr. med., Co-Präsident SCN; ${ }^{c}$ Prof. Dr. med., Vorstandsmitglied SCN; ${ }^{d}$ Dr. med., Co-Präsident SGMO und Vorstandsmitglied SCN; ${ }^{e}$ Dr. med., Vorstandsmit-

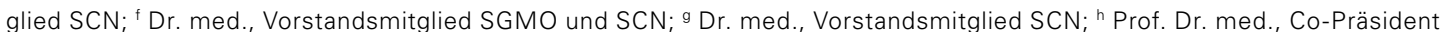
SGMO, Vorstandsmitglied SCN

Ausgangslage und Zielsetzung. Vor rund 10 Jahren haben in der Deutschschweiz grössere Kliniken begonnen, sich nach den Kriterien von ausländischen Gesellschaften zu zertifizieren. Zusätzlich entstanden erste organspezifische Zertifizierungen wie Brustzentren. Ein organübergreifendes Modell, welches unsere überwiegend dezentrale Versorgung durch freie Praxen und Spitalambulatorien berücksichtigt, stand sehr vielen Onkologinnen und Onkologen und ihren $\mathrm{Pa}$ tienten jedoch nicht zur Verfügung.
Im Rahmen des Nationalen Krebsprogramms 20112015 wollte die Schweizerische Gesellschaft für Medizinische Onkologie (SGMO) durch ein eigenes Zertifizierungsprogramm dazu beitragen, dass alle Patientinnen und Patienten Zugang zu einer wohnortnahen und qualitativ hochstehenden onkologischen Versorgung haben.

Vorgehen. Im Dezember 2012 hat die SGMO zusammen mit der Schweizerischen Gesellschaft für RadioOnkologie und Onkologiepflege Schweiz ein gemeinsa- 
mes Qualitätskonzept erarbeitet, dem die Mitglieder der SGMO grossmehrheitlich zugestimmt haben. In einem weiteren Schritt hat die SGMO den Verein Swiss Cancer Network (SCN) gegründet. Der SCN wurde u.a. damit beauftragt, ein Zertifizierungsprogramm auf Basis des Qualitätskonzepts zu entwickeln. Vor der Implementierung beurteilten die Mitglieder der SGMO das Programm erneut und verabschiedeten es im Januar 2014 mit einer Zustimmung von 87 Prozent.

Wofür das Zertifikat steht. Das Zertifikat SCN legt den Fokus auf evidenzbasierte Behandlungsempfehlungen unter Einschluss einer interdisziplinären und interprofessionellen Betreuung. Die einzuhaltenden Massnahmen verfolgen einen prozessorientierten Ansatz und respektieren die liberale, wettbewerbsorientierte Ausrichtung unseres Landes.

Die Zertifizierung umfasst fünf Dimensionen: Qualifikation des Fachpersonals; Form und Inhalt des Tumorboards inkl. Bericht; wissenschaftliche Grundlagen der individuellen Behandlung (sog. Leitlinien); Zusammenarbeit im Netzwerk von Pflege und Sozialinstitutionen; Zugang zu Behandlungsresultaten (detaillierte Informationen siehe www.sgmo.ch $\rightarrow$ Qualitätssicherung $\rightarrow$ Swiss Cancer Network).

Aktueller Stand der Zertifizierungen. Zurzeit sind in allen Landesteilen über 30 Institutionen von Einzelpraxen bis hin zu grösseren Spitalambulatorien gemäss Selbstdeklaration zertifiziert. Unter Beizug einer professionellen Auditgesellschaft und Einsatz von jeweils zwei Fachgutachtern (erfahrene und zu Auditoren geschulte Onkologen) wurden bisher fünf Institutionen vor Ort auditiert. Die SGMO erhöht nun stetig die Zahl der Audits.

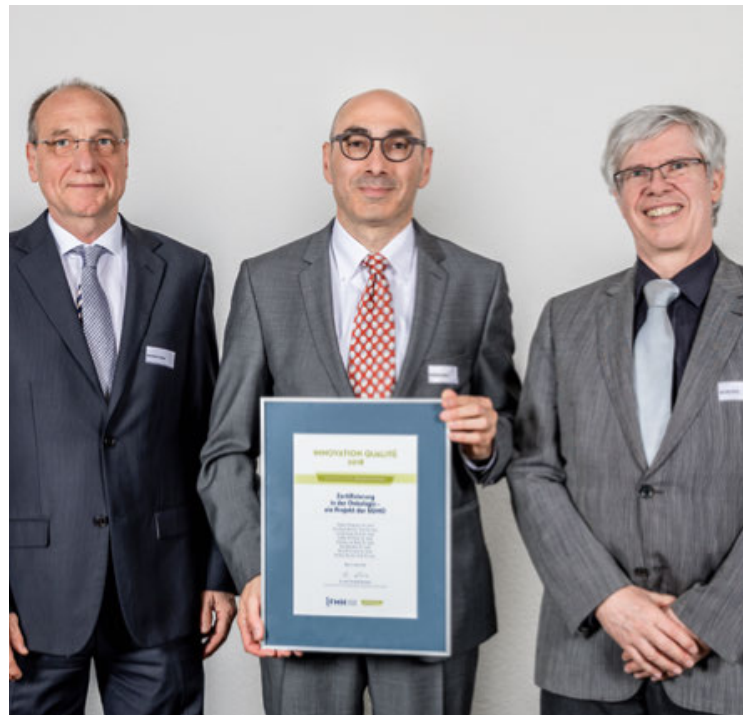

Volker Kirchner, Walter Mingrone und Kurt Beretta freuen sich über die Innovation Qualité.

Worauf wir stolz sind. Zu ihrer Freude ist es der SGMO aus eigener Initiative gelungen, gemeinsam mit Vertreterinnen aus privaten onkologischen Praxen, regionalen Spitalambulatorien, aber auch mit Kollegen von Universitätsspitälern ein Zertifizierungsprogramm zu schaffen, welches den Patientinnen und Patienten Gewähr für eine hochstehende onkologische Versorgung gibt. Zudem eines, das unseren nationalen Gegebenheiten und Traditionen der medizinischen Versorgung Rechnung trägt. Die SGMO wird die Zertifizierung in Diskussion und Absprache mit ihren Mitgliedern im Verlauf evaluieren und weiterentwickeln. 